\title{
Brucelosis humana y aborto
}

Dr. Ricardo Bechara Z.*

* Miembro Honorario de FECOLSOG

\section{DEFINICION Y GENERALIDADES}

Todos los autores conciden en definir la Brucelosis Humana como una enfermedad infecto-contagiosa transmitida al hombre por animales de la escala inferior tales como bovinos, equinos, porcinos y caprinos.

La Brucelosis Humana ha tenido una gama de definiciones que partiendo del nombre de Fiebre Mediterránea en 1.810, llegó hasta la denominación de enfermedad de las Cien Formas Clínicas en 1911 hasta anclar definitivamente en el rombre genérico de Brucelosis a secas.

El proceso más común de la transmisión de la Brucelosis a los humanos consiste en la ingestión de leche cruda proveniente de vacas enfermas, así como de la carne cruda o mal cocida y de vegetales contaminados con heces $u$ orina de animales infectados. Los más expuestos al contagio son los veterinarios, ordeñadores, matarifes y cuidadores de ganado vacuno, caballar, porcino y caprino. En nuestra casuística aparecen cuatro estudiantes de veterinaria que adquirieron la enfermedad por haberse expuesto a ello al tratar de extraer placenta retenida de vacas abortadoras, sin utilizar guentes de goma, pues se sabe que la piel escarificada o intacta no constituye barrera para evitar el contagio.

La brucelosis, sus receptáculos naturales y su peligrosidad para el hombre no se limitan a infecciones homólogas en los animales domésticos sino que se extiende a la infección cruzada entre los mismos y paso de la infección a otras especies que también pueden constituir peligro para la especie humana.

La mujer brucelósica puede dar a luz hijos sanos, especialmente si el embarazo ocurre después de la fase bacterémica, pero la in- fección se recrudece después del parto y el niño puede ser infectado por la ingestión de leche materna.

La enfermedad puede adquirirse por transfusión y por la inhalación de polvo de los mataderos y expendios de carne. Hay quien asegura que puede también adquirirse por medio de la saliva en el acto de besar. Lo único que falta por investigar es si el semen del hombre aloja o no a la Brucela, porque ya se sabe que la placenta de la mujer brucelósica si lo aloja y el germen es capaz de atravesar la barrera placentaria.

El período de incubación de la brucelosis varía entre 5 y 21 días y por estudios de alta credibilidad se sabe que el bacilo de la Brucela tiene una supervivencia así:

CUADRO No. 1

SUPERVIVENCIA DEL BACILO

MEDIO

SUPER VIVENCIA

EN DIAS

ORINA HUMANA

20

LECHE Y AGUA

60

POLVO DE LA CALLE Hasta

MANTEQUILLA

Hasta 10

VERDURAS

Hasta

CEBOLLAS

Hasta

HELADOS (LECHE CRUDA " $\quad 39$

QUESOS FRESCOS

15

La Brucelosis puede provocar amenorrea en la mujer y disminuye la líbido tanto en hombres como en mujeres. 
La mortalidad de la brucelosis es muy baja. En Estados Unidos llega al $1.2 \%$ y en la Isla de Malta sube a $2.3 \%$. No hay datos sobre muerte alguna por brucelosis en el Departamento de Córdoba (Colombia).

La brucelosis cauda la muerte por complicaciones como la endocarditis y la meningoencefalitis.

\section{HISTORIA}

La historia de la Brucelosis en Colombia se remonta al año 1927 cuando el desaparecido Profesor César Uribe Piedrahíta cultivó por primera vez el bacilo de la Brucelosis partiendo de placentas de vacas abortadoras de la Sabana de Bogotá.

En el Departamento de Córdoba el primero en detectar la existencia de la Brucelosis Humana fué el Dr. Héctor Espinosa Vellojín en el año 1952, al cultivar la sangre de un niño de 9 años que padecía accesos febriles y en quien los exámenes de laboratorio eran negativos para Tifoidea y Paludismo. Este diagnóstico fue comprobado en Bogotá a donde se envió sangre del infante enfermo y la confirmación fué realizada
Posteriormente el Dr. Rafael Espinosa Espinosa obtuvo su grado de Doctor en Medicina en la Universidad de Cartagena, presentando una tesis sobre Brucelosis Humana, en 1953.

\section{CUADRO No. 2}

\section{DATOS TESIS DE GRADO (1953)}

Especie

Humanos

Bovinos - Hembras

Bovinos - Toros

\section{Muestras}

$\%$ Posivos
La población ganadera del Departamento de Córdoba es de aproximadamente 4.000.000.oo de bovinos divididos tentativamente en mitad machos y mitad hembras y según esta estadística muy bien llevada en el Centro de Diagnósticos del Instituto Colombiano Agropecuario ICA, con sede en Montería, se obtuvieron los siguientes datos:

CUADRO No. 3

\section{DATOS DEL CENTRO DIAGNOSTICO ICA}

1973 - Octubre 1977

\section{ESPECIE}

BOVINOS

EQUINOS

PORCINOS

\section{LECHE (BOVINA)}

por el Profesor Luis Patiño Camargo en el Departamento de Medicina Tropical del Hospital San Juan de Dios. Según datos obtenidos el paciente en referencia cuidaba una cerda que había abortado varias veces, pero vale la pena anotar que el huésped intermediario no fué estudiado desde el punto de vista diagnóstico.

\section{POSITIVOS}

165

18
$\%$

19.05

11.00

15.00

Estos resultados nos están demostrando ampliamente y a las claras el potencial de peligrosidad que nos rodea y nos hace pensar que la enfermedad es más frecuente de lo que parece. El Dr. Luis Caraballo, Veterinario Jefe del Laboratorio de Investigaciones del ICA es uno de los preocupados por este problema de la Brucelosis Humana 
y es así como en estudio que realizó pudo comprobar que sobre 680 hombres y 750 mujeres controladas, obtuvo un índice del $10.5 \%$ de enfermos con 150 casos de positividad.

\section{EXPERIENCIA PERSONAL}

En los casos por nosotros observados y controlados diez años después, hemos podido comprobar que el brucelósico sigue siendo por el resto de su vida, pues encontramos que siempre presentaban una titulación que variaba entre el 1 x 20 al 1 × 40 .

¿Qué nos impulsó a pensar en la posibilidad de la probable relación de la Brucelosis Humana y el aborto? He aquí el suceso que nos sirvió de punto de partida: Eramos médicos habituales de una señora que vivía en una pequeña parcela cercana a Montería. Dicha señora contrajo matrimonio a la edad de 32 años y cuatro meses después de su enlace fuimos llevados de urgencias al lugar donde vivía y pudimos comprobar que presentaba un cuadro de aborto espontáneo irreversible. La paciente fué trasladada a Montería y en una Clínica le practicamos el vaciamiento del útero. Debemos anotar que la señora presentaba un estado febril agudo acompañado de escalofríos, cefalalgia, lasitud, raquialgia, sudoración profunda y malestar general. Coincidencialmente pudimos observar que en el corral vecino, en donde estaban encerrados sus bovinos había dos peones tratando de extraer sendas placentas a dos vacas que habían abortado y como sabemos que generalmente toda vaca que aborta es brucelósica, establecemos inmediatamente la posible relación que podría existir entre las enfermedades y el aborto de la paciente, amén de que esta nos había informado que ingería leche cruda y comía queso fabricado en su casa con leche sin hervir, proveniente de su pequeño hato. Procedimos a ordenar exámenes de laboratorio en relación con enfermedades febriles y para sorpresa nuestra resultó que la paciente tenía una titulación a Brucelosis del 1 x 820 de las 23 veces examinadas resultaron 13 positivas $56.52 \%$. Se prescribió el tratamiento de rigor consistente en 1 gramo diario de

tetraciclina y una ampolla diaria de estreptomicina de $1 \mathrm{grm}$. con lo que se obtuvo una remisión del cuadro anotado hasta llevar la titulación a 1 x 20. Posteriormente la señora tuvo una nueva gestación normal que finalizó en cesárea con la extracción de un niño con todas las características de absoluta normalidad.

De este episodio en adelante pudimos reunir 187 casos de brucelosis humana, todos comprobados con pruebas de laboratorio. El grupo está dividido en 138 hombres y 49 mujeres. Entre estas encontré 16 multíparas y 8 primigestantes con aborto de repetición, con brucelosis crónica y 6 mujeres con aborto durante el primer ataque agudo de la enfermedad.

CUADRO No. 4

\section{CASOS COMPROBADOS - MONTERIA}

Hombres

Mujeres

Total

Lo cierto en todo esto es que las multíparas habían tenido inicialmente embarazos normales con partos e hijos normales y e cuando adquirieron la enfermedad se le presentaron abortos de repetición los cuales cesaron cuando se les hizo el tratamiento de rigor y lograron volver a tener posteriormente embarazos normales con partos e hijos normales. Las primigestantes también tuvieron abortos repetidos que desaparecieron con el tratamiento y pudieron también tener posteriormente embarazos normales con hijos sanos. Los casos de las 16 multíparas y las 8 primigestantes a que nos referimos, presentaban brucelosis crónica con titulaciones que oscilaban entre 1 x 120 hasta 1 x 480 y fueron detectadas porque en el interrogatorio para confeccionar la historia clínica se pudo comprobar que habían tenido episodios febriles que no se debieron a paludismo ni tifoidea y que sin tratamiento alguno remitieron espontáneamente.

No aseguramos que la Brucelosis sea la res- 
CUADRO No. 5

PRIMIGESTANTES

8

6

\section{ABORTO}

MULTIPARAS

16

\section{REPETICION}

1o. ATAQUE
24 ponsable directa de los abortos en mujeres infectadas con brucelosis, pero tampoco podemos negarlo. Lo importante y muy llamativo es el hecho de coincidir la enfermedad con los abortos y especialmente el que después del tratamiento se haya logrado tener embarazos normales.

Solamente nos limitamos a verter nuestras experiencias y observaciones con la esperanza que otros médicos con más ánimo y más bríos puedan continuar el estudio de este tema que consideramos de singular importancia.

\section{RESUMEN}

Se presentan 187 casos de Brucelosis humana divididos en 138 hombres y 49 mujeres. De estas, hay 16 multíparas y 8 primigestantes con abortos de repetición, además de 6 mujeres con abortos en el curso de episodios agudos de Brucelosis. Se anota que efectuados los tratamientos del caso todas las mujeres afectadas de Brucelosis tuvieron posteriormente embarazos normales y los fetos nacieron en buenas condiciones.

En los 187 casos de Brucelosis tratados, no hubo ningún caso de muerte y parece que el tratamiento ha sido efectivo porque los pacientes se han mantenido clínicamente curados.

\section{HUMAN BRUCELLOSIS AND ABORTION}

\section{SUMMARY}

187 cases of human Brucellosis are shown, divided in 138 men and 49 women. From the latter, there are 16 multiparous and 8 first pregn. with repeated miscarriages, plus 6 women with miscarriages during acute episodes of Brucellosis.

It has to be emphasized that following the adequate treatment, all women with Brucellosis had normal pregnancies and the children were born in good condition. From the 187 cases treated for Brucellosis, none died, and it seems that the treatment was effective because the patients are clinically cured.

\section{REFERENCIAS}

Dr. Luis Caraballo - Conferencia.

De Lee Grinhill.- Principio y práctica de Obstetricia.

Dexeus.- Tratado de Obstetricia.

Cecil.- Textboock Of Medicine.

Cecil.- Loeb.- Tratado de Medicina Interna.

Th.v.Jaschke.- Tratado sobre Obstetricia.

Antonio Blanco Bautista y Lupo Ramos.- Tesis de grado.

Alfonso Romero Ochoa, Dagoberto Gómez A y Javier Parra V. Tesis de grado.

Daniel Gutiérrez y Fernando Pérez.- Tesis de grado. 